\title{
Plasma $n-3$ fatty acids and clinical outcomes in recent-onset rheumatoid arthritis
}

\author{
Susanna M. Proudman ${ }^{1,2 *}$, Leslie G. Cleland ${ }^{1,2}$, Robert G. Metcalf ${ }^{1}$, Thomas R. Sullivan ${ }^{3}$, \\ Llewellyn D. Spargo ${ }^{1}$ and Michael J. James ${ }^{1,2}$ \\ ${ }^{1}$ Rheumatology Unit, Royal Adelaide Hospital, Adelaide, SA 5000, Australia \\ ${ }^{2}$ Discipline of Medicine, University of Adelaide, Adelaide, SA 5000, Australia \\ ${ }^{3}$ Public Health (TRS), University of Adelaide, Adelaide, SA 5000, Australia
}

(Submitted 29 March 2015 - Final revision received 15 June 2015 - Accepted 22 June 2015)

\begin{abstract}
A randomised controlled trial (RCT) of high-dose $v$. low-dose fish oil in recent-onset rheumatoid arthritis (RA) demonstrated that the group allocated to high-dose fish oil had increased remission and decreased failure of disease-modifying anti-rheumatic drug (DMARD) therapy. This study examines the relationships between plasma phospholipid levels of the $n-3$ fatty acids in fish oil, EPA and DHA, and remission and DMARD use in recent-onset RA. EPA and DHA were measured in blood samples from both groups of the RCT. The data were analysed as a single cohort, and Cox proportional hazards models were used to examine relationships between plasma phospholipid (PL) EPA and DHA and various outcome measures. When analysed as a single cohort, plasma PL EPA was related to time to remission, with a one unit increase in EPA ( $1 \%$ total fatty acids) associated with a $12 \%$ increase in the probability of remission at any time during the study period (hazard ratio $(\mathrm{HR})=1.12 ; 95 \%$ CI 1.02, 1.23; $P=0.02$ ). Adjustment for smoking, anti-cyclic citrullinated peptide antibodies and 'shared epitope' HLA-DR allele status did not change the HR. Plasma PL EPA, adjusted for the same variables, was negatively related to time to DMARD failure (HR $=0.85 ; 95 \%$ CI 0.72, 0.99; $P=0.047$ ). The HR for DHA and time to remission or DMARD failure were similar in magnitude to those for EPA, but not statistically significant. Biomarkers of $n-3$ status, such as plasma PL EPA, have the potential to predict clinical outcomes relevant to standard drug treatment of RA patients.
\end{abstract}

Key words: Fish oil: Eicosapentaenoic acid: Docosahexaenoic acid: Arthritis: Nutritional immunology

Meta-analysis of studies with long-standing rheumatoid arthritis (RA) indicated that fish oil supplementation at doses supplying at least $2.9 \mathrm{~g} / \mathrm{d}$ of the $n-3$ fatty acids EPA and DHA decreased tender joint count and duration of morning stiffness ${ }^{(1,2)}$. Limitations of these studies include disease duration of 10-11 years and outdated drug regimens, which comprised sequential use of single-agent disease-modifying anti-rheumatic drug (DMARD) rather than combination therapy, and withdrawal of participants requiring dose or drug changes due to disease activity or drug toxicity ${ }^{(3)}$.

These limitations were addressed in a double-blind randomised controlled trial (RCT) of fish oil supplementation in patients with recent-onset RA where the median disease duration was 16 weeks $^{(4)}$. The RCT intervention was conducted within the framework of a standardised drug algorithm in which participants commenced triple DMARD therapy with methotrexate (MTX), sulphasalazine (SSZ) and hydroxychloroquine (HCQ), and dose escalation or drug changes were made according to rules that reflected the level of disease activity.
This allowed DMARD failure to be used as a primary outcome measure along with remission. There was an increased rate of remission and decreased rate of DMARD failure in the fish oil group at 1 year follow-up ${ }^{(4)}$.

The main $n$ - 3 fatty acids present in fish oil are EPA and DHA. They are precursors for the E- and D-resolvins and $\mathrm{PGD}_{2}$, which are involved in resolution of inflammation and can suppress production of both pro-inflammatory lipids such as $\mathrm{PGE}_{2}$ and leukotriene $\mathrm{B}_{4}$ and pro-inflammatory cytokines such as TNF- $\alpha$ and interleukin- $1 \beta^{(5-8)}$. Thus, it is plausible that tissue levels of EPA and/or DHA are responsible for the clinical effects observed in the RCT of fish oil in recent-onset $\mathrm{RA}^{(4)}$. Plasma levels of $n$ - 3 fatty acids obtained from fish oil consumption are highly variable between individuals even under clinical trial conditions with good compliance ${ }^{(9)}$. Moreover, the proportionate increase in plasma EPA with fish oil ingestion is much larger than that for DHA and they have very different turnover times, resulting in tissue levels of DHA being much higher than those of EPA without ingestion of fish or

Abbreviations: ACR, American College of Rheumatology; DAS28, Disease Activity Score; DMARD, disease-modifying anti-rheumatic drug; HR, hazard ratio. PL, phospholipid; RA, rheumatoid arthritis; RCT, randomised controlled trial.

* Corresponding author: S. M. Proudman, fax: +61 88222 5895, email susanna.proudman@health.sa.gov.au 
fish oil ${ }^{(10-13)}$. This can lead to considerable overlap of both plasma EPA and DHA levels between control and fish oil intervention groups in parallel group RCTs, limiting the ability of intention-to-treat analysis to assess the relationship between the clinical effects of fish oil and the plasma levels of $n-3$ fatty acids $^{(9)}$. The purpose of this study was to examine the relationships between the outcome measures of remission and DMARD failure with plasma biomarkers of fish oil supplementation - EPA and DHA.

\section{Methods}

\section{Subjects}

Consecutive patients who attended the Early Arthritis Clinic at the Royal Adelaide Hospital were screened. Those who satisfied the inclusion criteria for active RA that met the 1987 revised American College of Rheumatology (ACR) criteria, with disease duration $<12$ months and no previous treatment with DMARD, were enrolled into a RCT with a fish oil intervention arm. The study was approved by the Royal Adelaide Hospital Research Ethics Committee, and participants gave their informed consent. The Australian New Zealand Clinical Trials Registration number is ACTRN12613000579796. The complete inclusion and exclusion criteria have been described ${ }^{(4)}$.

\section{Fish oil treatment}

For the RCT, subjects were allocated 2:1 to receive high-dose or low-dose fish oil. The high-dose group was instructed to take $10 \mathrm{ml} / \mathrm{d}$ fish oil concentrate (BLT Incromega TG3525) providing $3.2 \mathrm{~g} \mathrm{EPA}+2.3 \mathrm{~g} \mathrm{DHA} / \mathrm{d}$ as TAG, and the low-dose group was instructed to take $10 \mathrm{ml} / \mathrm{d}$ sunola oil:capelin oil (2:1) providing $0 \cdot 21 \mathrm{~g} \mathrm{EPA}+0 \cdot 19 \mathrm{~g} \mathrm{DHA} / \mathrm{d}$ as TAG. The 1 year results of the trial have been reported ${ }^{(4)}$.

\section{Protocol and drug treatment}

After enrolment to the RCT, subjects were seen every 3 weeks for the first 12 weeks and then every 6 weeks thereafter. At each visit, clinical and biochemical assessments of disease activity were performed and blood was sampled for fatty acid measurements. The assessments of disease activity included twenty-eight joint tender and swollen joint counts, erythrocyte sedimentation rate (ESR) and determination of C-reactive protein levels, which were used to determine whether there should be drug escalation according to pre-defined rules ${ }^{(14)}$. All the subjects commenced treatment on triple DMARD therapy comprising SSZ $0.5 \mathrm{~g} / \mathrm{d}$, HCQ $200 \mathrm{mg}$ twice per d (bd) and MTX $10 \mathrm{mg}$ once per week SSZ was increased by $0.5 \mathrm{~g} / \mathrm{d}$ at weekly intervals to $1 \mathrm{~g}$ bd. HCQ dose remained unchanged. MTX was increased only when there was active disease above a pre-defined threshold during the scheduled clinic visits (Fig. 1). The criteria for the level of disease activity that triggered drug dose escalation have been described and it was determined that they equated to a Disease Activity Score (DAS28) $\geq 3 \cdot 2^{(4)}$. The DAS28 score is a composite of tender and swollen joint counts for twenty-eight joints, ESR and general health score ${ }^{(15)}$. A DAS28 score of 3.2 is considered to be minimal disease activity. If the disease activity was not above this threshold, then the MTX dose was held constant. If MTX reached the maximum allowable dose of $25 \mathrm{mg} /$ week, then it was considered to be a case of 'DMARD failure' and leflunomide $10 \mathrm{mg} / \mathrm{d}$ was introduced (Fig. 1).

\section{Outcome measures}

DMARD failure, as measured by progression to leflunomide treatment, was used as a measure of disease control by the drugs used according to the algorithm described above.

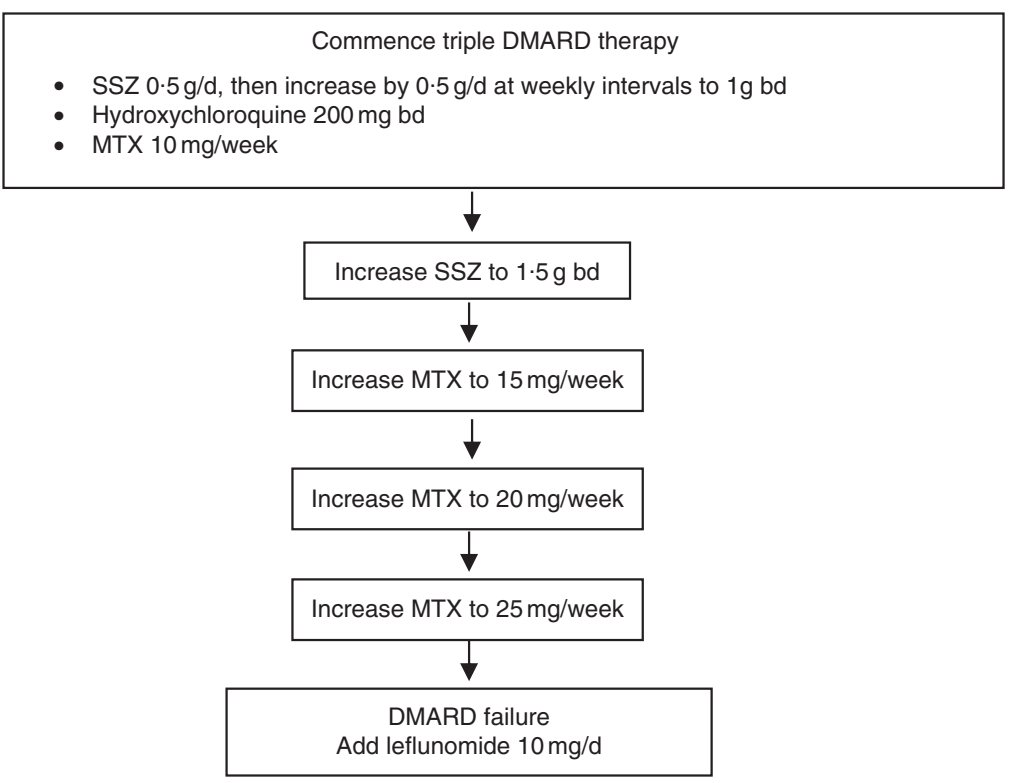

Fig. 1. Drug treatments allowed by the rules-based algorithm. Disease activity was assessed at each clinic visit and drug escalation occurred if the disease activity exceeded a pre-specified threshold described in the text. DMARD, disease-modifying anti-rheumatic drugs; SSZ, sulfasalazine; bd, twice per d; MTX, methotrexate. 
Remission was assessed by two standards - (a) the ACR criteria for remission and (b) a DAS28-ESR score $<2 \cdot 6$, which is the accepted cut-off for DAS28 remission ${ }^{(16)}$. ACR remission is the more stringent assessment and is considered to be complete absence of measurable disease ${ }^{(17)}$. However, there frequently is residual disease activity in those with DAS28 scores $<2 \cdot 6$, although it is called remission.

\section{Fatty acid measurement}

Blood was collected into EDTA-containing tubes at clinic visits. The number of clinic visits in 12 months varied between subjects according to how well their disease was controlled. The mean number of blood samples per subject from which fatty acids were measured was 5.4. The plasma fraction was removed and extracted fresh with isopropanol-chloroform (1:2). The chloroform layer was stored at $-20^{\circ} \mathrm{C}$ until analysis, which was typically within 1-2 months. Phospholipids (PL) were separated by TLC, subjected to methanolysis and the fatty acids methyl esters were determined by GLC as we have described previously ${ }^{(18)}$

\section{Analyses}

The original RCT group allocations were ignored and the subjects were treated as a single cohort. The analyses sought to determine whether there were relationships between plasma PL EPA or DHA and to determine the following outcome measures: time (from randomisation) to DMARD failure, to ACR remission and to DAS28 remission. Univariate and multivariate Cox proportional hazards models were fitted to the data. The strength of these models is the ability to incorporate timevarying covariates. Therefore, mean values of plasma PL EPA or DHA over the year were not used, but rather PL EPA and DHA were treated as time-varying covariates with their measurements updated at each follow-up appointment. Proportionality assumption was tested and appeared reasonable in all models. Quadratic terms for EPA and DHA were not statistically significant in any of the models. After obtaining an unadjusted hazard ratio (HR) for plasma PL EPA or DHA (model 1), the baseline confounders, smoking history, plasma anti-cyclic citrullinated protein antibodies (anti-CCP) and the genetic $H L A-D R \beta 1$ risk marker, known commonly as the 'shared epitope', were added to the model. These measures and markers have prognostic value and they were included as covariates in the original analysis of the $\mathrm{RCT}^{(4)}$.

\section{Results}

\section{Subjects}

Subjects had recent-onset active RA with median disease duration of 16 weeks and mean DAS28 score of $5 \cdot 7$ (Table 1).

\section{Disease outcomes}

The first occurrences of ACR and DAS28 remission were at 3 weeks after commencement of treatment, and the events accumulated in a linear manner throughout the year (Fig. 2). At 1 year, the overall ACR and DAS28 remission rates were 33 and
Table 1. Demographics and clinical characteristics relevant to participants' rheumatoid arthritis

(Mean values and standard deviations; median values and interquartile ranges (IQR), numbers and parcentages; $n$ 139)

\begin{tabular}{|c|c|c|c|}
\hline & $n$ & & $\%$ \\
\hline \multicolumn{4}{|c|}{ Age at disease onset (years) } \\
\hline Mean & & $55 \cdot 8$ & \\
\hline SD & & $15 \cdot 2$ & \\
\hline Female & 101 & & $72 \cdot 7$ \\
\hline \multicolumn{4}{|l|}{ BMI $\left(\mathrm{kg} / \mathrm{m}^{2}\right)$} \\
\hline Median & & $27 \cdot 8$ & \\
\hline IQR & & $23 \cdot 4-31 \cdot 5$ & \\
\hline \multicolumn{4}{|c|}{ Duration of polyarthritis (weeks) } \\
\hline Median & & 16 & \\
\hline IQR & & $12-24$ & \\
\hline RF positive & 84 & & $60 \cdot 4$ \\
\hline Anti-CCP positive & 74 & & $53 \cdot 2$ \\
\hline Shared epitope positive & 90 & & $64 \cdot 7$ \\
\hline Never smoked & 54 & & 38.8 \\
\hline \multicolumn{4}{|l|}{ DAS28 } \\
\hline Mean & & 5.7 & \\
\hline SD & & $1 \cdot 2$ & \\
\hline
\end{tabular}

RF, rheumatoid factor; anti-CCP, antibodies to cyclic citrullinated peptides; DAS28, Disease Activity Score at baseline, a composite score swollen and tender joint counts of twenty-eight joints, blood inflammatory markers, and patient global health.

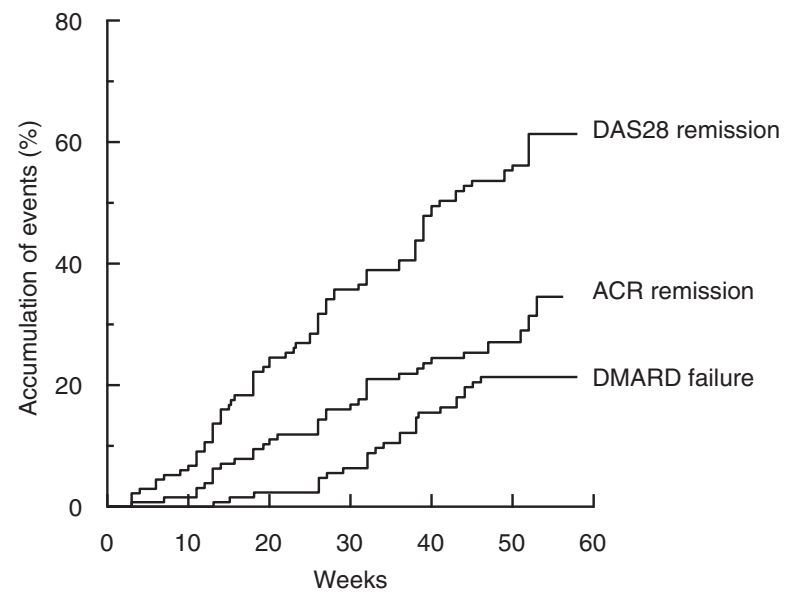

Fig. 2. Time to event expressed as the percentage achieving remission, as defined by the American College of Rheumatology criteria (ACR remission) or according to the composite Disease Activity Score of twenty-eight joints (DAS28 remission) or percentage failing triple therapy with Disease-Modifying Anti-Rheumatic Drugs (DMARD).

$61 \%$, respectively. The first occurrence of DMARD failure and commencement of leflunomide occurred at 13 weeks, and by 1 year the cumulative rate was $21 \%$ (Fig. 2).

\section{Fatty acids}

Combining the high-dose and low-dose fish oil groups from the RCT provided a range of plasma PL EPA values from 0.4 to $10 \cdot 5$ and DHA values from 1.9 to $9.9 \%$ of total fatty acids. In general, the EPA and DHA values from subjects in the high-dose fish oil group were higher compared with the low-dose group, but there was considerable overlap (Fig. 3). 


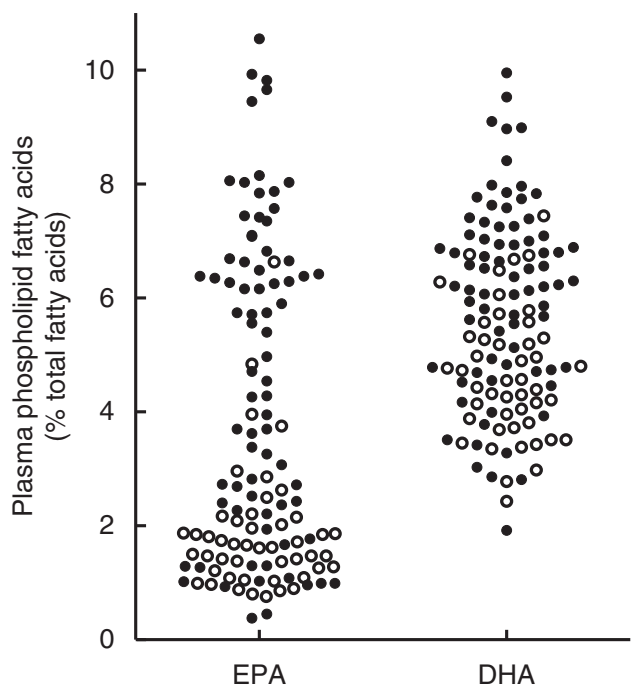

Fig. 3. Mean plasma phospholipid levels of EPA and DHA averaged over 1 year with the exclusion of the baseline values in the total cohort of recent-onset rheumatoid arthritis patients who had participated in a randomised controlled trial with a 2:1 random allocation to high-dose or low-dose fish oil ${ }^{(4)}$ $\bigcirc$, Low-dose fish oil; $\mathbf{O}$, high-dose fish oil.

\section{Relationships between fatty acids and disease outcomes}

EPA was positively associated with time to ACR remission in univariate analysis such that a one unit increase in plasma PL EPA (as \% of total fatty acids) was associated with a $12.2 \%$ increase in the probability of ACR remission at any time during the study period $(\mathrm{HR}=1.12 ; 95 \%$ CI $1.02,1.23 ; P=0.02)$ (Table 2). This was not altered by correction for the baseline characteristics of smoking, anti-CCP antibodies and shared epitope. EPA, adjusted for the same variables, was negatively related to time to DMARD failure $(\mathrm{HR}=0.85 ; 95 \% \mathrm{CI} 0.73,0.99$; $P=0.047)$. The HR for DHA and time to ACR remission or DMARD failure were numerically similar to those for EPA, but not statistically significant. Plasma PL EPA + DHA was positively associated with time to ACR remission, and, although the association with time to DMARD failure was in the positive direction, it was not quite statistically significant $(\mathrm{HR}=0.92$; $95 \%$ CI $0 \cdot 84,1 \cdot 01 ; P=0 \cdot 08)$. There was no relationship between $\mathrm{EPA}$ or DHA or EPA + DHA and time to DAS28 remission. There was no relationship between arachidonic acid/EPA and any of the outcome measures (Table 2).

\section{Discussion}

A double-blind, RCT with these participants demonstrated that the fish oil supplementation group had decreased failure of DMARD therapy and increased ACR remission at 1 year followup with no effect on DAS28 remission ${ }^{(4)}$. The RCT used a fish oil concentrate and aimed for ingestion of $5.5 \mathrm{~g} / \mathrm{d}$ EPA + DHA. Compliance monitoring indicated that the achieved intake was $3.7 \mathrm{~g} / \mathrm{d}$ EPA + DHA, which exceeded the intake most likely to demonstrate an anti-inflammatory effect, which is $2.9 \mathrm{~g} / \mathrm{d}^{(2,19)}$. Although the by-group analysis of RCT results is useful, it is limited as it provides no information on target levels of intake.
Table 2. Relationship between plasma phospholipid $n-3$ fatty acids and disease outcomes

(Hazard ratios and $95 \%$ confidence intervals)

\begin{tabular}{|c|c|c|c|}
\hline Variables & Hazard ratio & $95 \% \mathrm{Cl}$ & $P$ \\
\hline \multicolumn{4}{|l|}{ ACR remission } \\
\hline \multirow[t]{2}{*}{ EPA } & $1 \cdot 12$ & $1.02,1.23$ & 0.02 \\
\hline & $1 \cdot 12^{*}$ & $1.02,1.24$ & 0.02 \\
\hline \multirow[t]{2}{*}{$\mathrm{DHA}$} & $1 \cdot 12$ & $0.95,1.32$ & 0.17 \\
\hline & $1 \cdot 10^{*}$ & $0.93,1.31$ & 0.27 \\
\hline \multirow[t]{2}{*}{$\mathrm{EPA}+\mathrm{DHA}$} & 1.07 & $1.01,1.14$ & 0.04 \\
\hline & $1.07^{*}$ & $1.00,1.14$ & 0.05 \\
\hline \multirow[t]{2}{*}{ AA/EPA } & 0.99 & $0.93,1.06$ & 0.77 \\
\hline & $1.00^{*}$ & $0.93,1.06$ & 0.92 \\
\hline \multicolumn{4}{|c|}{ DAS28 remission } \\
\hline \multirow[t]{2}{*}{ EPA } & 1.03 & $0.95,1.12$ & 0.47 \\
\hline & $1.03^{*}$ & $0.95,1.12$ & 0.47 \\
\hline \multirow[t]{2}{*}{$\mathrm{DHA}$} & 1.03 & $0.91,1.17$ & 0.65 \\
\hline & $1.03^{*}$ & $0.90,1.18$ & 0.65 \\
\hline \multirow[t]{2}{*}{$\mathrm{EPA}+\mathrm{DHA}$} & 1.02 & $0.97,1.07$ & 0.51 \\
\hline & $1.02^{*}$ & $0.97,1.07$ & 0.51 \\
\hline \multirow[t]{2}{*}{ AA/EPA } & 0.99 & $0.95,1.04$ & 0.83 \\
\hline & $0.99^{*}$ & $0.95,1.04$ & 0.77 \\
\hline \multicolumn{4}{|l|}{ Drug failure } \\
\hline \multirow[t]{2}{*}{ EPA } & 0.86 & $0.73,1.00$ & 0.051 \\
\hline & $0.85^{\star}$ & $0.73,0.99$ & 0.047 \\
\hline \multirow[t]{2}{*}{$\mathrm{DHA}$} & 0.89 & $0.73,1.10$ & 0.29 \\
\hline & $0.89^{*}$ & $0.72,1.10$ & 0.29 \\
\hline \multirow[t]{2}{*}{$\mathrm{EPA}+\mathrm{DHA}$} & 0.92 & $0.84,1.01$ & 0.09 \\
\hline & $0.92^{*}$ & $0.84,1.01$ & 0.08 \\
\hline \multirow[t]{2}{*}{ AA/EPA } & 1.03 & $0.98,1.08$ & 0.22 \\
\hline & $1.04^{*}$ & $0.98,1.10$ & 0.17 \\
\hline
\end{tabular}

ACR, American College of Rheumatology; AA, arachidonic acid; DAS28, Disease Activity Score.

* Corrected for smoking, anti-CCP and shared epitope.

$\dagger$ Failure of disease-modifying anti-inflammatory drugs signified by commencement of leflunomide.

The rationale behind the study of fish oil in RA is that EPA and DHA can suppress inflammatory mediator production and are precursors of molecules that can resolve inflammation ${ }^{(5-8)}$. It has been demonstrated that supplementation with fish oil increased plasma or serum concentrations of $\mathrm{E}$ - and D-resolvins, but greater increases were found for their monohydroxy precursors 17-hydroxy DHA and 18-hydroxy EPA ${ }^{(20,21)}$. If these are among the mechanisms behind the benefits of fish oil in RA, the tissue and/or blood levels of EPA and DHA are most likely related to outcomes, and knowledge of these could be useful for implementation of the RCT findings.

The present analysis exploited the range of plasma PL EPA and DHA values generated by the high-dose and low-dose fish oil groups of the RCT. Higher plasma PL EPA levels were associated with a higher likelihood of ACR remission and a lower likelihood of failure of triple DMARD therapy. The HR for plasma PL DHA and the same outcome measures were numerically similar to those for EPA but were not statistically significant. Possibly, this is a result of a narrower range of plasma PL DHA compared with plasma PL EPA values as seen in Fig. 3. Participants in the low-dose fish oil group of the RCT generally had low plasma PL EPA, but not low plasma PL DHA levels. This is a common finding, which may be due to the slower turnover of DHA compared with $\mathrm{EPA}^{(10,12,13)}$. Plasma PL EPA and DHA levels were highly correlated with each other (Spearman's $r 0.87$; $95 \%$ CI 0.81, 0.91; $P<0.0001$ ), and therefore the observed associations of EPA and DHA with the clinical 
outcomes may not reflect the true independent effects of DHA and EPA due to potential confounding.

The lack of association of plasma PL EPA with DAS28 remission was congruent with the lack of effect of fish oil on DAS28 remission in the RCT. In both cases, there were positive effects of fish oil ingestion or plasma PL EPA levels on ACR remission but not on DAS28 remission. The reasons for this difference are not known, but ACR and DAS28 measures do not reflect the same level of disease suppression. DAS28 remission is defined as DAS28 $<2.6$ and reflects low disease activity as well as remission. Accordingly, the proportions of this cohort achieving DAS28 remission and the more stringent ACR remission were 61 and $33 \%$, respectively. Moreover, the sample variation around the mean DAS28 scores was large ${ }^{(4)}$.

The HR of 1.12 for EPA and time to ACR remission can be interpreted as the likelihood of ACR remission at any time during the study period being increased by $12 \%$ for a one unit increase in EPA, which is an increase in plasma PL EPA of $1 \%$ of total fatty acids. A $1.1 \%$ increase in plasma PL EPA has been achieved with ingestion of fish oil containing $1.5 \mathrm{~g} \mathrm{EPA}$ and $1.8 \mathrm{~g} \mathrm{DHA} / \mathrm{d}$ on $2 \mathrm{~d} /$ week and this was considered the equivalent of two meals per week of fatty fish such as salmon $^{(22)}$. Although most other fish would not provide these amounts of EPA+DHA, it is an indication that elevation of plasma PL EPA is achievable with fish even if higher levels are likely to be obtained with daily fish oil supplementation.

All RA patients at our clinics receive advice to take $10-15 \mathrm{ml}$ fish oil per day, and uptake of this advice ranges from nil to partial to complete ${ }^{(14)}$. The results of this study demonstrate the potential to use plasma biomarkers such as EPA levels to make predictions about outcomes in clinical practice with RA, and this could be used to give advice on target levels to patients. The importance of this is that the plasma levels of EPA and DHA correlated poorly with dose $\mathrm{e}^{(9-13)}$. Feasibility of such an approach is demonstrated by the application of erythrocytes $\mathrm{EPA}+\mathrm{DHA}$ as a risk factor for ischaemic heart disease ${ }^{(23)}$, and this is now a test offered by commercial providers in the USA.

\section{Acknowledgements}

This study was supported by the National Health Medical Research Council of Australia and Royal Adelaide Hospital Research Committee. Melrose Health has provided support for ongoing studies.

S. M. P., L. G. C. and M. J. J. were responsible for study design, enrolment and/or treatment of study subjects, data interpretation, writing and critical manuscript review; T. R. S. carried out data analysis, data interpretation and critical manuscript review; L. D. S. and R. G. M. contributed towards data collation/checking, data interpretation and critical manuscript review.

The authors declare that they have no conflicts of interest.

\section{References}

1. Fortin PR, Lew RA, Liang MH, et al. (1995) Validation of a meta-analysis: the effects of fish oil in rheumatoid arthritis. J Clin Epidemiol 48, 1379-1390.
2. Proudman SM, Cleland LG \& James MJ (2008) Dietary omega-3 fats for treatment of inflammatory joint disease: efficacy and utility. Rheum Dis Clin North Am 34, 469-479.

3. James MJ, Proudman SM \& Cleland LG (2010) Fish oil and rheumatoid arthritis: past, present and future. Proc Nutr Soc 69, 316-323.

4. Proudman SM, James MJ, Spargo LD, et al. (2015) Fish oil in recent onset rheumatoid arthritis: a randomised, double-blind controlled trial within algorithm-based drug use. Ann Rheum Dis 74, 89-95.

5. Calder PC (2013) Omega-3 polyunsaturated fatty acids and inflammatory processes: nutrition or pharmacology? Br J Clin Pharmacol 75, 645-662.

6. Serhan CN, Chiang N \& Van Dyke TE (2008) Resolving inflammation: dual anti-inflammatory and pro-resolution lipid mediators. Nat Rev Immunol 8, 349-361.

7. James MJ, Gibson RA \& Cleland LG (2000) Dietary polyunsaturated fatty acids and inflammatory mediator production. Am J Clin Nutr 71, Suppl., 343S-348S.

8. Lawrence $\mathrm{T} \&$ \& Gilroy DW (2007) Chronic inflammation: a failure of resolution? Int J Exp Pathol 88, 85-94.

9. James MJ, Sullivan TR, Metcalf RG, et al. (2014) Pitfalls in the use of randomised controlled trials for fish oil studies with cardiac patients. Br J Nutr 112, 812-820.

10. Zuijdgeest-van Leeuwen SD, Dagnelie PC, Rietveld T, et al. (1999) Incorporation and washout of orally administered $n-3$ fatty acid ethyl esters in different plasma lipid fractions. $\mathrm{BrJ}$ Nutr 82, 481-488.

11. Metcalf RG, James MJ, Gibson RA, et al. (2007) Effects of fishoil supplementation on myocardial fatty acids in humans. AmJ Clin Nutr 85, 1222-1228.

12. Brown AJ, Pang E \& Roberts DC (1991) Persistent changes in the fatty acid composition of erythrocyte membranes after moderate intake of $n-3$ polyunsaturated fatty acids: study design implications. Am J Clin Nutr 54, 668-673.

13. Cao J, Schwichtenberg KA, Hanson NQ, et al. (2006) Incorporation and clearance of omega-3 fatty acids in erythrocyte membranes and plasma phospholipids. Clin Chem 52, 2265-2272.

14. Proudman SM, Keen HI, Stamp LK, et al. (2007) Response-driven combination therapy with conventional disease-modifying antirheumatic drugs can achieve high response rates in early rheumatoid arthritis with minimal glucocorticoid and nonsteroidal anti-inflammatory drug use. Semin Arthritis Rheum 37, 99-111.

15. Fransen J \& van Riel PL (2005) The Disease Activity Score and the EULAR response criteria. Clin Exp Rheumatol 23, Suppl. 39, S93-S99.

16. Makinen $\mathrm{H}$, Kautiainen $\mathrm{H}$, Hannonen $\mathrm{P}$, et al. (2005) Is DAS28 an appropriate tool to assess remission in rheumatoid arthritis? Ann Rheum Dis 64, 1410-1413.

17. Pinals RS, Masi AT \& Larsen RA (1981) Preliminary criteria for clinical remission in rheumatoid arthritis. Arthritis Rheum 24, 1308-1315.

18. Metcalf RG, Mantzioris E, Cleland LG, et al. (2003) A practical approach to increasing intake of $n-3$ fats: use of novel foods enriched with $n-3$ fats. Eur J Clin Nutr 57, 1605-1612.

19. Cleland LG, James MJ \& Proudman SM (2006) Fish oil: what the prescriber needs to know. Arthritis Res Ther 8, 202.

20. Barden A, Mas E, Croft KD, et al. (2014) Short-term n-3 fatty acid supplementation but not aspirin increases plasma proresolving mediators of inflammation. J Lipid Res $\mathbf{5 5}$, 2401-2407.

21. Mas E, Croft KD, Zahra P, et al. (2012) Resolvins D1, D2, and other mediators of self-limited resolution of inflammation in 
human blood following $n-3$ fatty acid supplementation. Clin Chem 58, 1476-1484.

22. Browning LM, Walker CG, Mander AP, et al. (2012) Incorporation of eicosapentaenoic and docosahexaenoic acids into lipid pools when given as supplements providing doses equivalent to typical intakes of oily fish. Am J Clin Nutr 96 $748-758$.

23. Harris WS \& Von Schacky C (2004) The Omega-3 Index: a new risk factor for death from coronary heart disease? Prev Med 39, 212-220. 